\title{
The Fornax dwarf galaxy as a remnant of recent dwarf-dwarf merging in the Local Group
}

\author{
C. Yozin and K. Bekki \\ ICRAR, M468, The University of Western Australia 35 Stirling Highway, Crawley \\ Western Australia, 6009, Australia
}

\begin{abstract}
We present results from the first numerical analysis to support the hypothesis, first proposed in Coleman et al. (2004), that the Fornax dwarf galaxy was formed from the minor merging of two dwarfs about 2 Gyr ago. Using orbits for the Fornax dwarf that are consistent with the latest proper motion measurements, our dynamical evolution models show that the observed asymmetric shell-like substructures can be formed from the remnant of a smaller dwarf during minor merging. These models also predict the formation of diffuse stellar streams. We discuss how these stellar substructures depend on model parameters of dwarf-dwarf merging, and how the intermediate-age subpopulations found in the vicinity of these substructures may be formed from gas accretion in the past merger events. We also suggest that one of Fornax's GCs originates from a merged dwarf companion, and demonstrate where as yet undetected tidal streams or HI gas formed from the dwarf merging may be found in the outer halo of the Galaxy.
\end{abstract}

Subject headings: galaxies: kinematics and dynamics — galaxies: individual (Fornax) - Local Group

\section{Introduction}

The Fornax galaxy possesses many unusual characteristics that have warranted far greater investigation than might be expected for a typical dwarf spheroidal (dSph). Lying in the Local Group at a distance of $138 \mathrm{kpc}$ (Saviane et al. 2000) and receding at $53.3 \mathrm{kms}^{-1}$ (Walker et al. 2007), the foremost distinguishing features are six Globular Clusters (GC), resulting in one of the highest known specific frequencies of all galaxies (29, from van den Bergh 1998). Although these GCs are associated with Fornax (Mateo et al. 1991), with distances to the galaxy center ranging between 0.24 and $1.60 \mathrm{kpc}$, there exists an atypical 
spread in metallicity and age (Buonanno et al. 1999, and Strader et al. 2003). Moreover, dynamical friction acting on the GCs in the dark matter (DM) dominated Fornax should have resulted in their assimilation into the Fornax nucleus since their formation more than 10 Gyr ago. Tidal interaction with the Milky Way (MW) has been invoked to explain the latter by Oh et al. (2000); further studies by Goerdt et al. (2006) propose that a cored DM halo would increase the effective sinking time for GCs, while Cowsik et al. (2009) asserts dynamical friction to be weaker than previously thought.

The above discussion lies in parallel but separate from recent photometric analyses by Coleman et al. (2004, 2005). These studies have interpreted statistically-significant $(4.5 \sigma)$ stellar overdensities as evidence of an extant shell substructure, with the most prominent shell 'sheets' aligned with the major axis of Fornax and extending beyond the tidal radius $\left(r_{t}=71 \pm 4\right.$ arcmin, Irwin \& Hatzidimitriou 1995) of Fornax . Tidal interaction with the MW is discarded as a causative factor, since the predominantly intermediate (2 Gyr) age of the overdensity subpopulation contrasts with the otherwise mixed aged general population of Fornax (Buonanno et al. 1999, Stetson et al. 1998). Quinn (1984) showed that low-energy minor mergers can result in shell-like substructures, and a gas-rich companion galaxy may also provide the fuel source for a past starburst, given the present lack of $H_{I}$ gas observed in Fornax. Olszewski et al. (2006) argue however that the metallicity in the overdensities bear the signature of Fornax itself.

In both debates, the influence of the Milky Way (MW) potential has been contemplated with respect to the assumed orbit of Fornax. Successive measurements for the present day proper motion of Fornax have been conducted recently by Dinescu et al. (2004), Piatek et al. (2007), Walker et al. (2008), and Méndez et al. (2011), hereafter D04, P07, W08 and M11 respectively. M11 gives a high-energy orbit with an orbital period $\sim 21$ Gyr, while D04, P07 and W08 imply a low-energy orbit with orbital periods ranging between 5-8 Gyr. In all cases, Fornax is conceived to have been at perigalacticon in the last $~ 100-300$ Gyr. Méndez et al. (2011) propose that this triggered an enhanced star formation at this period, and perhaps a similarly induced starburst >10 Gyr ago, thus supporting their hyper-extended orbit (if the merger scenario can explain the remaining intermediate subpopulation). By contrast, the minimum galactocentric radial distance of $131-148 \mathrm{kpc}$ leads Coleman et al. (2005) to assert the relative insignificance of tidal forces at this separation. To date, no numerical studies have been conducted to assert the interaction and merger history of Fornax, using the latest measurements of its proper motion.

The purpose of this LETTER is to test the hypothesis of Coleman et al. (2004) that Fornax is a remnant of recent dwarf merging. We show that the overdensities are evidence of a shell substructure formed by the most recent merger event. We also discuss how the 
recent dwarf merging is important for (i) the formation of intermediate-age ( $\sim 2$ Gyr) stars in the core of Fornax and (ii) physical properties of its unusual globular cluster system. We make predictions for future observations that would constrain the present range of proper motions and further support the merger hypothesis.

\section{The model}

Our investigation assumes that the present day Fornax dSph is the product of the merger of two dwarfs, referred to hereafter as Fornax A (host) and B (companion) respectively. Our merger model is developed in two stages: first, we evaluate the orbital history of Fornax for a given Galactic potential and a mass model for Fornax A/B (§2.1) by using the "backward integration scheme" used in similar simulations (e.g., Diaz \& Bekki 2012, hereafter DB12). In the second step $(\S 2.2)$ we introduce an collisionless N-body representation of Fornax B, and investigate its dynamical evolution under the gravitational influence of the Galaxy and Fornax A.

\subsection{Orbit Integration}

We adopt the same Galaxy model as used in our previous simulations on the evolution of the Magellanic Cloud (DB12). The dominant component is the extended halo, for which we adopt a NFW density distribution (Navarro et al. 1996), up to a virial radius $R_{v i r}$ of 175kpc:

$$
\rho_{N F W}(r)=\frac{\rho_{0}}{\left(c r / R_{v i r}\right)\left(1+c r / R_{v i r}\right)^{2}},
$$

from which the virial mass $M_{v i r}$ is derived as:

$$
M_{v i r}=4 \pi \rho_{0}\left(R_{v i r} / c^{3}\right)(\ln (1+c)-c /(1+c))=1.30 \times 10^{12} M_{\odot}
$$

where $r$ is the spherical radius, $\rho_{0}$ is the characteristic density and $c$ is a concentration parameter $(=12)$. The disk uses the potential of Miyamoto \& Nagai (1975), which has the form:

$$
\Phi_{\text {disk }}=-\frac{G M_{d}}{\sqrt{R_{2}+\left(a+\sqrt{z_{2}+b_{2}}\right)^{2}}},
$$

where the total mass of the disk $M_{d}=5.0 \times 10^{10} M_{\odot}$ (Binney \& Tremaine 2008), $R=$ $\sqrt{x^{2}+y^{2}}$, and $a$ and $b$ are scale parameters with values $3.5 \mathrm{kpc}$ and $0.35 \mathrm{kpc}$ respectively. Finally, the Galactic bulge uses the spherical potential model of Hernquist (1990):

$$
\Phi_{\text {bulge }}=-\frac{G M_{b}}{r+c_{b}}
$$


where the mass of the bulge $M_{b}$ is $0.5 \times 10^{12} M_{\odot}$ and $c_{b}=0.7 \mathrm{kpc}$ (Binney \& Tremaine 2008). The total mass of the MW, up to a limit radius of $300 \mathrm{kpc}$, is thus calculated as $\sim 1.73 \times 10^{12} M_{\odot}$. Fornax $\mathrm{A}$ is assumed to have a Plummer potential:

$$
\Phi_{F A}(r)=-\frac{G M_{F A}}{\sqrt{r_{2}+a_{F A}{ }^{2}}},
$$

where $M_{F A}$ is the total mass of $\left(10^{9} M_{\odot}\right.$, consistent with the latest observations (e.g., de Boer et al. 2011), and $a_{F A}$ is a softening radius of $0.6 \mathrm{kpc}$. We consider the spherical Plummer model sufficient for the slightly oblate extant Fornax dSph; Hernquist \& Quinn (1989) show a complex dependence of the shell structure alignment on primary galaxy potential, but we consider this dependence sufficiently small to ignore within the scope of this preliminary study.

The time integration of the equation of motion is performed by using 2nd-order leap-frog method (Murai \& Fujimoto 1980) with a time step interval of $\sim 1.4 \times 10^{6}$ yr. For initial space velocities, we used the proper motions of P07 and M11 as representative of the lowand high-energy orbits respectively. The calculated error in the measurements of P07 were sufficiently low to justify the use of space velocities (in the galactocentric rest frame) derived from the nominal proper motion. The measurements of M11 by contrast are accompanied by significant confidence limits, in which case we separately considered the nominal (M11), lowest- (M11L) and highest- $(\mathrm{M} 11 \mathrm{H})$ energy combinations from this data to attain the corresponding space velocities $\left(V_{X}, V_{Y}, V_{Z}\right)$ in Table 1 . We use the coordinate system adopted in DB12, in which Fornax is currently located at position $(X, Y, Z)=(-39.0,-48.0,-126.0)$ kpc, as derived from van den Bergh (1999). For each orbit, the integration scheme is utilised until the time $\left(T_{M}\right)$ at which a merger is judged to have commenced. To establish the time varying dependence of our postulated mergers, and accommodate the age range of young stars in Fornax (e.g. Fig.4, Tsujimoto 2011), we vary the merger time among models from $T_{M}=-3.5$ to -2 Gyr, where $T=0$ is the present time.

\section{2. $\quad$ N-Body model and merger parameters}

Fornax B is assumed to be a dwarf disk galaxy embedded in a massive dark matter halo, and is therefore represented here by N-body particles in a self-consistent manner. We adopt the dwarf disk model adopted in our previous simulations on dynamical evolution of dwarf disk galaxies around the Galaxy, the details of which are given in Bekki \& Yong (2012).

The total mass of Fornax B $\left(M_{\mathrm{t}, \mathrm{dw}}\right)$ is assumed as $5 \%$ of Fornax A (i.e., $M_{\mathrm{t}, \mathrm{dw}}=$ $\left.5 \times 10^{7} M_{\odot}\right)$. The observed average metallicity of Fornax is consistent with the merger of 
two satellites (Fornax A and B) in a 20:1 stellar mass ratio assuming that the two merging satellites conform to the luminosity-metallicity relationship of MW dSph satellites (e.g., Kirby et al. 2011). The density profile of the dark matter halo with a total mass of $M_{\mathrm{dm}, \mathrm{dw}}$ is represented by that proposed by Salucci \& Burkert (2000):

$$
\rho_{\mathrm{dm}}(r)=\frac{\rho_{\mathrm{dm}, 0}}{\left(r+a_{\mathrm{dm}}\right)\left(r^{2}+a_{\mathrm{dm}}^{2}\right)},
$$

where $\rho_{\mathrm{dm}, 0}$ and $a_{\mathrm{dm}}$ are the central dark matter density and the core (scale) radius, respectively. The mass-ratio of $M_{\mathrm{dm}, \mathrm{dw}}$ to $M_{\mathrm{t}, \mathrm{dw}}$ is set to be 0.9 , while $a_{\mathrm{dm}}$ is varied for different models. The stellar component of the dwarf is modelled as a bulge-less stellar disk with the total mass of $M_{\mathrm{s}, \mathrm{dw}}$ and the size of $R_{\mathrm{s}, \mathrm{dw}}$. The radial $(R)$ and vertical $(Z)$ density profiles of the stellar disk are assumed to be proportional to $\exp \left(-R / R_{0}\right)$ with scale length $R_{0}=0.2 R_{\mathrm{s}, \mathrm{dw}}$ and to $\operatorname{sech}^{2}\left(Z / Z_{0}\right)$ with scale length $Z_{0}=0.04 R_{\mathrm{s}, \mathrm{dw}}$, respectively. The gravitational softening length for the dark matter and stellar disk is set to be $2.7 \mathrm{pc}$.

In addition to the rotational velocity caused by the gravitational field of disk and dark halo components, the initial radial and azimuthal velocity dispersions are assigned to the disc component according to the epicyclic theory with Toomre's parameter $Q=1.5$. The mass-ratio of $M_{\mathrm{s}, \mathrm{dw}}$ to $M_{\mathrm{t}, \mathrm{dw}}$ is set to be 0.1 . The spin of the dwarf galaxy is specified by two angles $\theta$ and $\phi$ (in units of degrees). Here, $\theta$ is the angle between the $z$-axis and the vector of the angular momentum of the disk, and $\phi$ is the azimuthal angle measured from the $x$-axis to the projection of the angular momentum vector of the disk onto the $x$ - $y$ plane. The initial position of Fornax B, with respect to Fornax A, is described with:

$$
\mathbf{X}_{A B}=\left(r_{A B} \cos (\Phi), 0, r_{A B} \sin (\Phi)\right)
$$

where $r_{A B}$ is the initial radial separation and $\Phi$ is an inclination angle; similarly, the velocity is:

$$
\mathbf{V}_{A B}=\left(f_{r} V_{c}, f_{t} V_{c}, 0\right),
$$

where $V_{c}$ is the circular velocity at $r_{A B}$, and $f_{r}$ and $f_{t}$ are radial and tangential component factors respectively. The locations of the overdensities from Coleman et al. (2004, 2005) would appear to describe an aligned shell, which is most often imposed by radial or nearradial collisions (Hernquist \& Quinn 1989). The relative sparseness of the Local Group, and Fornax's location on the periphery of those satellites considered bound to the Galaxy, would result in galaxy interactions tending towards those observed in field rather than cluster environments. We thus constrained the trajectory of Fornax B with respect to A to a low-energy, infalling parabolic orbit, with $f_{r}=-0.5$ and $f_{t}=0.1$. The morphology of a shell substructure is also sensitive to the initial orientation of the encounter: the sharp-edged features of the shell are phase-wrapped sheets as seen edge-on, with their overall distribution 
(the symmetry plane) seen face-on. We assumed that the overdensities fit this perspective, and thus we determined $\theta=135, \phi=0$ and $\Phi=30^{\circ}$, which together promote a face-on view from a heliocentric location at $T=0$.

The dynamical evolution of Fornax B was investigated for a wide extent of the contingent parameter space described by the variables here defined, with models consisting of 200,000 particles (split equally between the stellar and DM components). For parameter sets where the stellar distribution is comparable with extant observations, we confirm the results with higher resolution 1,000,000 particle models. All simulations are implemented with an original code (e.g., Bekki 2011), which executes with the parallel computing architecture CUDA, on a PC cluster installed with Nvidia GTX580 GPUs at the University of Western Australia.

\section{Results}

Figure 1 shows the backwards integrated orbits of Fornax A for the nominal proper motions of P07 and M11, and conveys the wide discrepancy in their respective proximity to the MW. For a merger instantiated at $\left(T_{M}=-3.5\right)$ using the proper motion of P07 we find, in the dynamic stellar distribution, overdensities are developed for a specific parameter subset of Fornax B that we define as Model 1 (Table 1). The top row of Figure 2 gives the projected stellar distribution of Model 1, with a Gaussian-convolved image of Fornax from Coleman et al. (2005) for comparison; the outer overdensity is highlighted together with the inferred axis of symmetry. At $T=-1.4$, a classical type 1 (Prieur 1990 classification) 'bowtie' structure is prominent. The regions of maximal stellar density are further elucidated by first dividing the on-sky distribution projection into quadrants, and then counting the number of stars that lie within each equal width bin of radius from Fornax A. Accordingly, the top-left panel of Figure 3 shows salient maximal densities at $1^{\circ}$ to $1.5^{\circ}$ arranged in opposing quadrants, analogous to Coleman et al. (2005). As in other studies (e.g. Weil et al 1996), these peaks exist briefly ( 0.1 Gyr) before diminishing through mixing. Fornax $\mathrm{B}$ is not completely disrupted, but we find it to first reside at the maximal stellar density region, before later descending to Fornax A's stellar core. Notably, we find a bimodality in the heliocentric velocity of the Fornax B stellar remnant up to a radius of $\sim 0.7^{\circ}$; this can be compared with a similar bimodality observed for the metal-poor stars of the Fornax dSph (Battaglia et al. 2006), as conveyed in the right-side panels of Fig. 3).

When an otherwise identical model is run but with $T_{M}=2.1$ Gyr (Model 2), the anticipated overdensities are in fact not reproduced at $T=0$. Tidal influence on Fornax is deemed strongest therefore during its approach to perigalacticon, and to recover overdensities at $T=0$ for this $T_{M}$, Fornax $\mathrm{B}$ is required to be more compact in its original state. With 
a decreased disk $R_{\mathrm{s}, \mathrm{dw}}$ and halo core radius $a_{\mathrm{dm}}$, Model 3 is conveyed in Fig. 2 and Fig. 3 to possess a distinct overdense remnant (the surviving stellar core of Fornax B) at $T=0$, which is again comparable qualitatively with Fornax. Taking a broader view of the stellar distribution, the left-side panels of Figure 4 show a tidal stream in Models 1 and 3, which extends up to a length of $\sim 30^{\circ}$ in alignment with the prior orbit of Fornax, and for a further $\sim 20^{\circ}$ in the opposing direction.

In contrast, our models with high energy orbits (M11, M11L and M11H) develop largely axisymmetric shell structures (Type 2), as conveyed by the representative Model 4 (with proper motion M11) of Fig. 2 (lower-right). This variation from Models 1-3 is attributed to the lessened capacity of the Galaxy to lock Fornax B into a more radial orbit and accretion by its host. These models have no tidal streams; in the case where tidal influence is most endorsed, with M11L and a lesser initial density of Fornax B (Model 5), small tidal plumes do develop (Fig. 4, lower-right panel).

\section{Discussion and Conclusions}

In this letter, we have demonstrated that the Fornax galaxy could be the remnant of a recent merger. We believe that Models 1 and 3 define two bounding cases, where a shell substructure, qualitatively similar to that observed in the Fornax dSph, is achieved for a merger occurring between $T_{M}=-3.5$ and $T_{M}=-2.1$ Gyr ago. Future photometry of these proposed shell regions may reveal their full distribution and thus allow further fine

tuning of the merger time (and the initial density Fornax B); at this stage, however, the merger hypothesis put forward by Coleman et al. (2004) appears valid. Furthermore, the morphology of this structure may be indicative of the orbital motion of Fornax; we anticipate the discovery of more substructure, but the current observations are most commensurate with the low-energy orbit of P07 (implying also D04, W08). We have additionally predicted tidal structure, similar to the well-studied stellar streams of the Sagittarius dSph (MartińezDelgado et al. 2004). Future observations of extant tails can provide constraints therefore on the proper motion of Fornax. We suggest further that the maximal density regions of the substructure could represent the core of the companion, which has been not completely disrupted by the merger and subsequent relaxation. We intend that the models derived here feed a more detailed investigation, with emphasis on the gaseous evolution of the companion and a more accurate model for the potential field of Fornax.

The stellar tails will likely be accompanied by an extensive dispersal of gas; the subsequent infall back to the core occurs over a timescale (Hernquist \& Spergel 1992, Hibbard \& Mihos 1995) that far exceeds the time since our postulated merger. In spite of the significant 
disruption to the disk, the original stellar core of the companion survives in our simulations. While a rapid segregation of stars and gas may occur in an isolated galaxy (Weil \& Hernquist 1993), the tidal action on the remnant distribution may make gaseous infall less efficient, and thus the shell regions would have exhibited the merger-induced starburst rather than the galactic nuclei. Photometric analysis of stars within the inner and outer overdensities (Coleman et al. 2004, 2005) reveal a small age range centered on 2 Gyr; this truncation of star formation may be associated with the quick exhaustion of this cold gas under the action of tidal shocks, or instead a rapidly decreasing gas density (Kojima \& Noguchi 1997). Where infall does occur, the distinct velocity bimodality common to both the simulated Fornax B remnant and the metal-poor subpopulation of the Fornax dSph may support the concept of a young subpopulation recently introduced to the otherwise aged Fornax dSph.

Our merger scenario may also add to the discussion on Fornax's globular clusters. Firstly, Buonanno et al. (1999) state a dichotomy in age between GCs with H4 being uniquely younger than clusters H1, H2, H3 and H5 by 3 Gyr. This contrasts with the opinion of Strader et al. (2003) where H5 is several years the youngest. In either case, the age discrepancy may be resolved by the accretion of the younger GC initially in the companion galaxy. Based on observations of Forbes and Bridges (2011), several of the Galactic GCs have been traced to the Fornax-Leo-Sculptor system, and therefore may have recently been part of this companion; the tidal streams we have predicted may contain further GCs as evidence of this. Secondly, the dynamical heating attributable to a merger event (Kazantzidis et al. 2008) could excite the orbits of the GCs. Therefore it is possible that the GCs have not since sunken to the core of the host and coalesced into its nucleus due to the past merger events of the Fornax dSph. Oh et al. (2000) calculated the timescale for mass accretion to the galaxy core (without tidal influences), as $\sim 1$ Gyr, therefore implying that this heating mechanism may be required at several instances in the past. While this may appear pre-

sumptuous, it naturally supports the common observation that star formation in Fornax has occurred in a sequence of discontinuous starbursts.

We gratefully acknowledge the suggestions of the anonymous referee that improved this manuscript.

\section{REFERENCES}

Battaglia, G. et al. 2006, A\&A, 459, 423

Bekki, K. 2011, MNRAS, 416, 2359 
Bekki, K. \& Yong, D. 2012, MNRAS, 419, 2063

Binney, J. \& Tremaine, S. 2008, Galactic Dynamics, Princeton Univ. Press (Princeton, NJ)

Buonanno, R. et al., 1999, AJ, 118, 1671

Coleman, M. G. et al., 2004, AJ, 127, 832

Coleman, M. G. et al. 2005, AJ, 129, 1443

Cowsik, R. et al., 2009, ApJ, 699, 1389

Dinescu, D. I. et al., 2004, AJ, 128, 687

Diaz, J. \& Bekki, K. 2012, MNRAS, 413, 2015

Forbes, D. A. \& Bridges, B., 2011, MNRAS, 404, 1203

Goerdt, T. et al., 2006, MNRAS, 368, 1073

Hernquist, L. \& Quinn, P., 1989, ApJ, 342, 1

Hernquist, L., 1990, ApJ, 356, 359

Hernquist, L. \& Spergel, D. N. 1992, ApJ, 399, L117

Hibbard, J. E. \& Mihos, J. C. 1995, AJ, 110, 140

Irwin, M., \& Hatzidimitriou, D. 1995, MNRAS, 277, 1354

Kazantzidis, S et al., 2008, ApJ, 688, 254

Kirby, E. N. et al., 2011, ApJ, 727, 79

Kojima, M. \& Noguchi, M. 1997, ApJ, 481, 132

Martińez-Delgado, D. et al., 2004, ApJ, 601, 242

Mateo, M. et al., 1991, AJ, 102, 914

Méndez, R. A., Costa, E., Gallart, C. et al., 2011, AJ, 142, 93

Miyamoto, M. \& Nagai, R. 1975, PASJ, 27, 533

Murai, T., \& Fujimoto, M. 1980, PASJ, 32, 581

Navarro, J., F. et al., 1996, ApJ, 490, 493 
Oh, K. S. et al., 2000, AJ, 531, 727

Olszewski, E. W., Mateo, M., \& Harris, J., et al., 2006, AJ, 131, 912

Piatek, S. et al., 2007, AJ, 133, 818

Prieur, J. -L. 1990, in Dynamic and Interactions of Galaxies, ed. R. Wielen (Springer-Verlag, Berlin)

Quinn, P. 1984, ApJ, 279, 596

Salucci, P. \& Burkert, A. 2000, ApJ, 312, L27

Saviane, I. et al., 2000, A\&A, 355, 56

Stetson, P.B. et al. 1998, PASP, 110, 533

Strader, J. et al., 2003, AJ, 125, 1291

Tsujimoto, T. 2011, ApJ, 736, 113

van den Bergh, A. 1998, ApJ, 492, 41

van den Bergh, S, 1999, in IAU Symp. 192, The Stellar Content of Local Group Galaxies, ed. P. Whitelock \& R. Cannon (Cambridge: Cambridge Univ. Press), 3

Walker, M. G. et al., 2007, ApJ, 667, L53

Walker, M. G. et al., 2008, ApJ, 688, L75

Weil, M. L. \& Hernquist, L., 1993, ApJ, 405, 142

Weil, M. L. et al., 1996, ApJ, 490, 664 


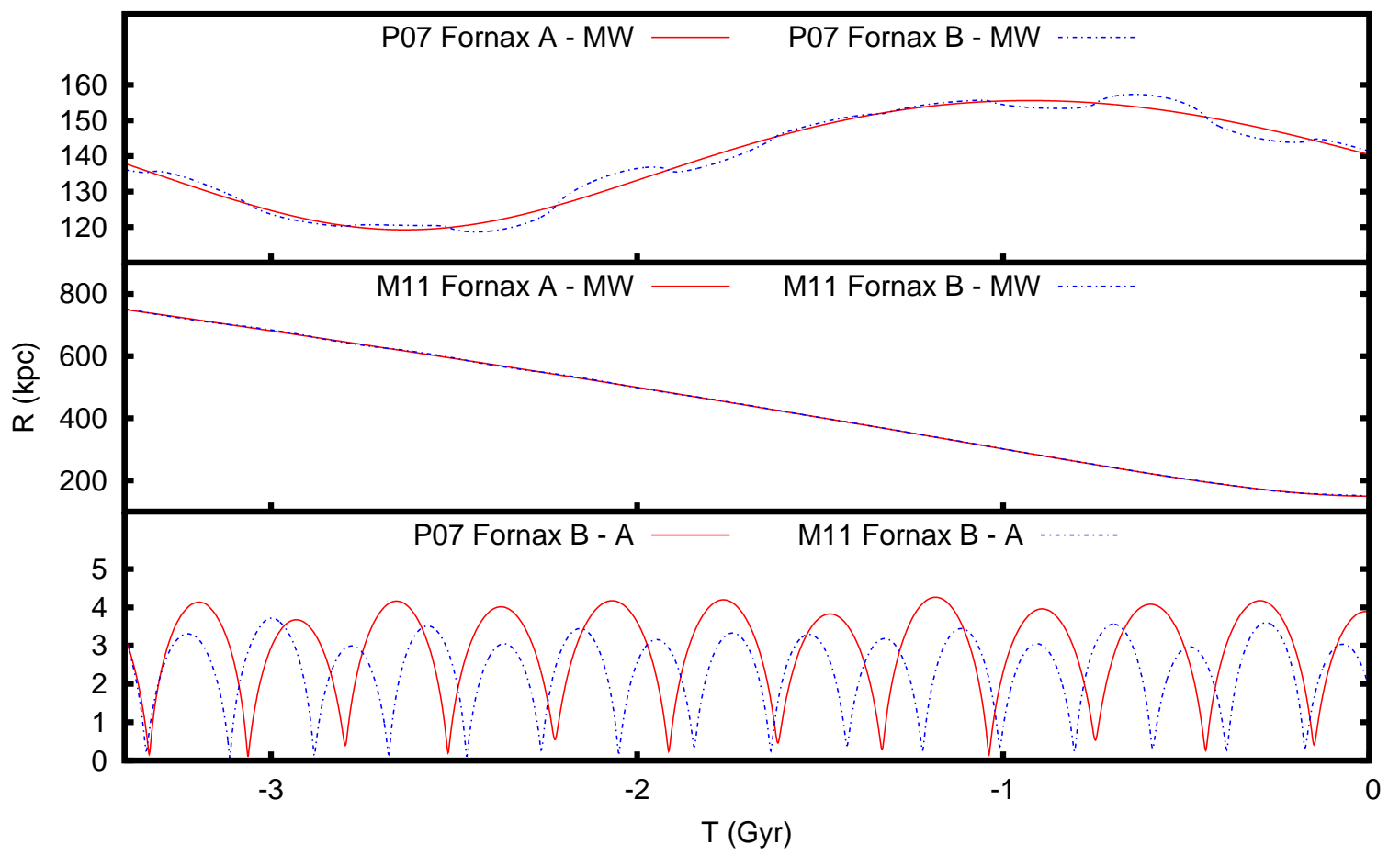

Fig. 1. - The radial distance between Fornax A and MW (red), Fornax B and MW (blue) for P07 (top panel) and M11 (middle panel); the lower panel shows the radial distance between Fornax A and B for P07 (Red) and M11 (blue).

Table 1. Summary of model parameters

\begin{tabular}{lcccccccc}
\hline \hline Model & Proper Motion & $\begin{array}{c}T_{M} \\
G y r\end{array}$ & $\begin{array}{c}V_{X} \\
k m s^{-1}\end{array}$ & $\begin{array}{c}V_{Y} \\
k m s^{-1}\end{array}$ & $\begin{array}{c}V_{Z} \\
k m s^{-1}\end{array}$ & $\begin{array}{c}r_{A B} \\
k p c\end{array}$ & $\begin{array}{c}a_{\mathrm{dm}} \\
k p c\end{array}$ & $\begin{array}{c}R_{\mathrm{s}, \mathrm{dw}} \\
k p c\end{array}$ \\
\hline 1 & $\mathrm{P} 07$ & -3.5 & -137 & -80 & 103 & 3.75 & 0.645 & 0.6 \\
2 & $\mathrm{P} 07$ & -2.1 & -137 & -80 & 103 & 3.75 & 0.645 & 0.6 \\
3 & $\mathrm{P} 07$ & -2.1 & -137 & -80 & 103 & 3.75 & 0.43 & 0.4 \\
4 & $\mathrm{M} 11$ & -3.5 & -226 & -185 & 149 & 3.75 & 0.645 & 0.6 \\
5 & $\mathrm{M} 11 \mathrm{~L}$ & -3.5 & -125 & -96 & 99 & 3.75 & 0.645 & 0.6 \\
- & $\mathrm{M} 11 \mathrm{H}$ & - & -326 & -274 & 199 & - & - & - \\
\hline
\end{tabular}




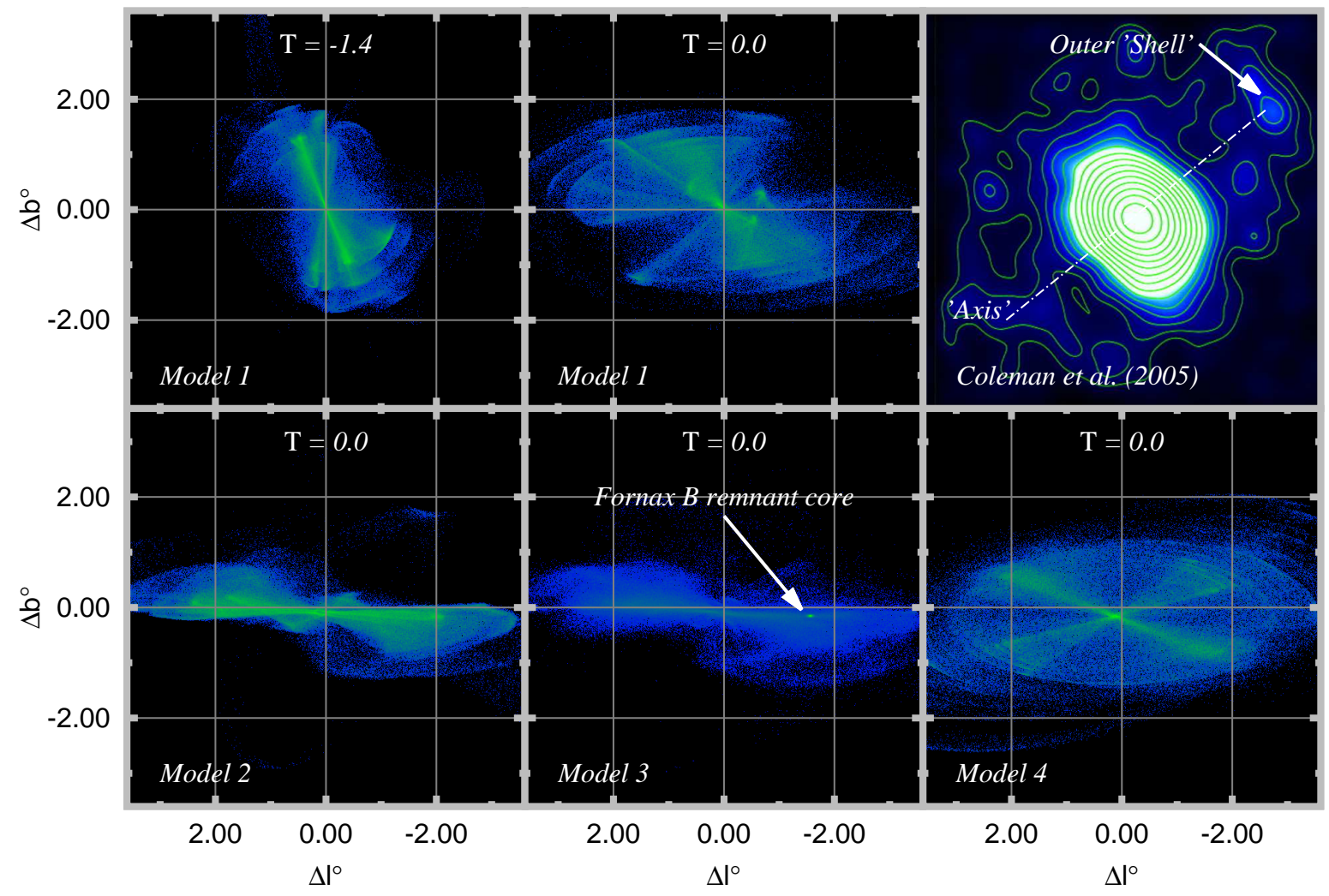

Fig. 2.- The stellar distribution of Fornax B as projected in galactic spherical coordinates and centered on the location of Fornax A. The time $\mathrm{T}$ (in units of Gyr) is shown at the top of each panel. The colour scale in each panel relates to the cumulative number of stars within each pixel of the 600x600 resolution image, for Model 1 (top-left, top-center), Model 2 (lower-left), Model 3 (lower-middle), and Model 4 (lower-right). For comparison, the Gaussian-convolved RGB stellar distribution of Fornax (Figure 23 of Coleman et al. 2005) is given in the top-right. 

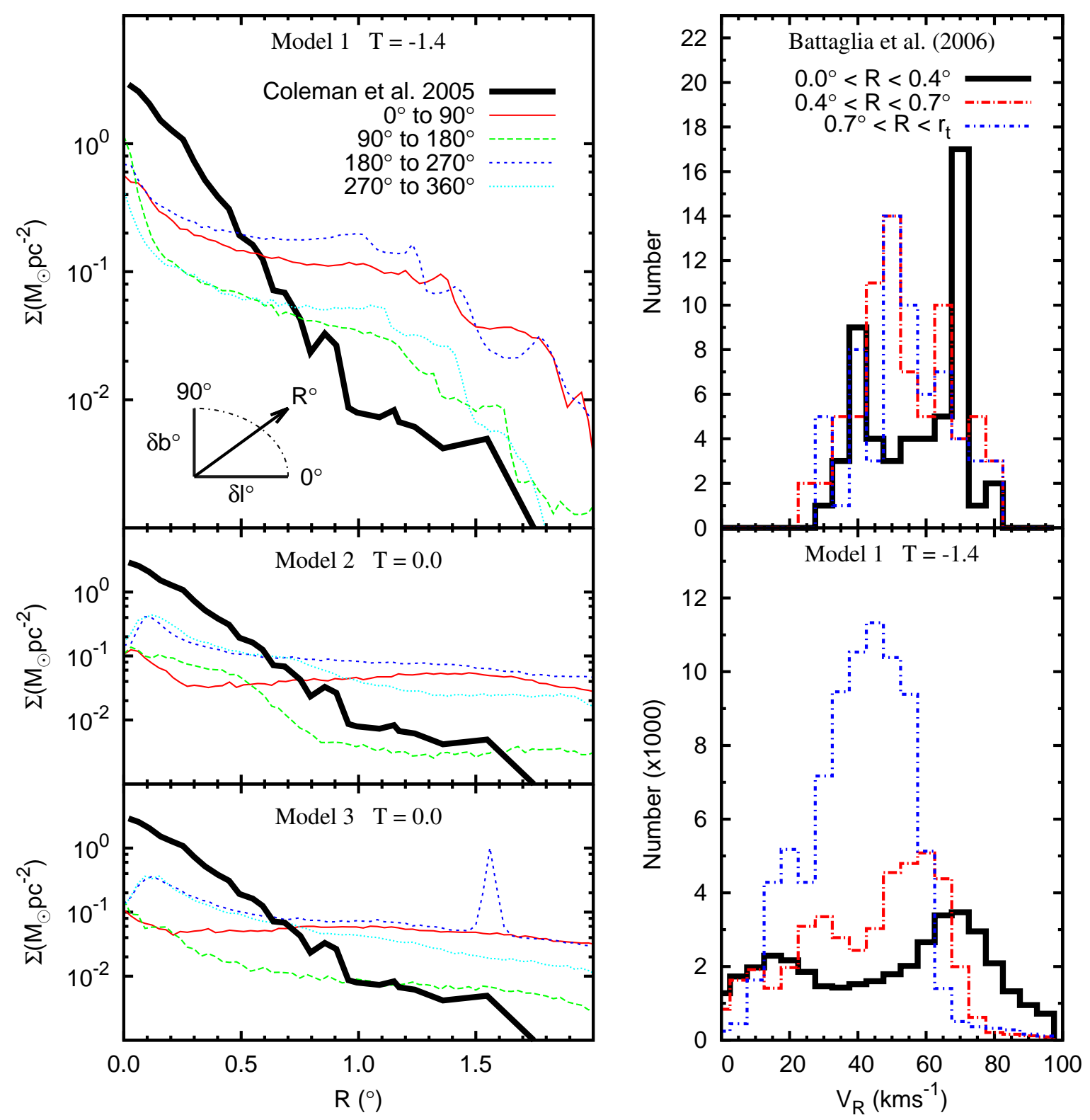

Fig. 3.- (Left) The stellar (mass) surface density of Fornax B for Models 1, 2 and 3, compared with stellar (number) density data (reproduced from Coleman et al. 2005). The distribution of stars from each N-body simulation is divided between four on-sky quadrants centered on Fornax A, at time T (in units of Gyr). (Right) The star count with Heliocentric Velocity for stars within distance bins up to the Fornax tidal radius, $r_{t}$ for (top) observational data reproduced from Battaglia et al. (2006), and (bottom) Model 1 from this work. 

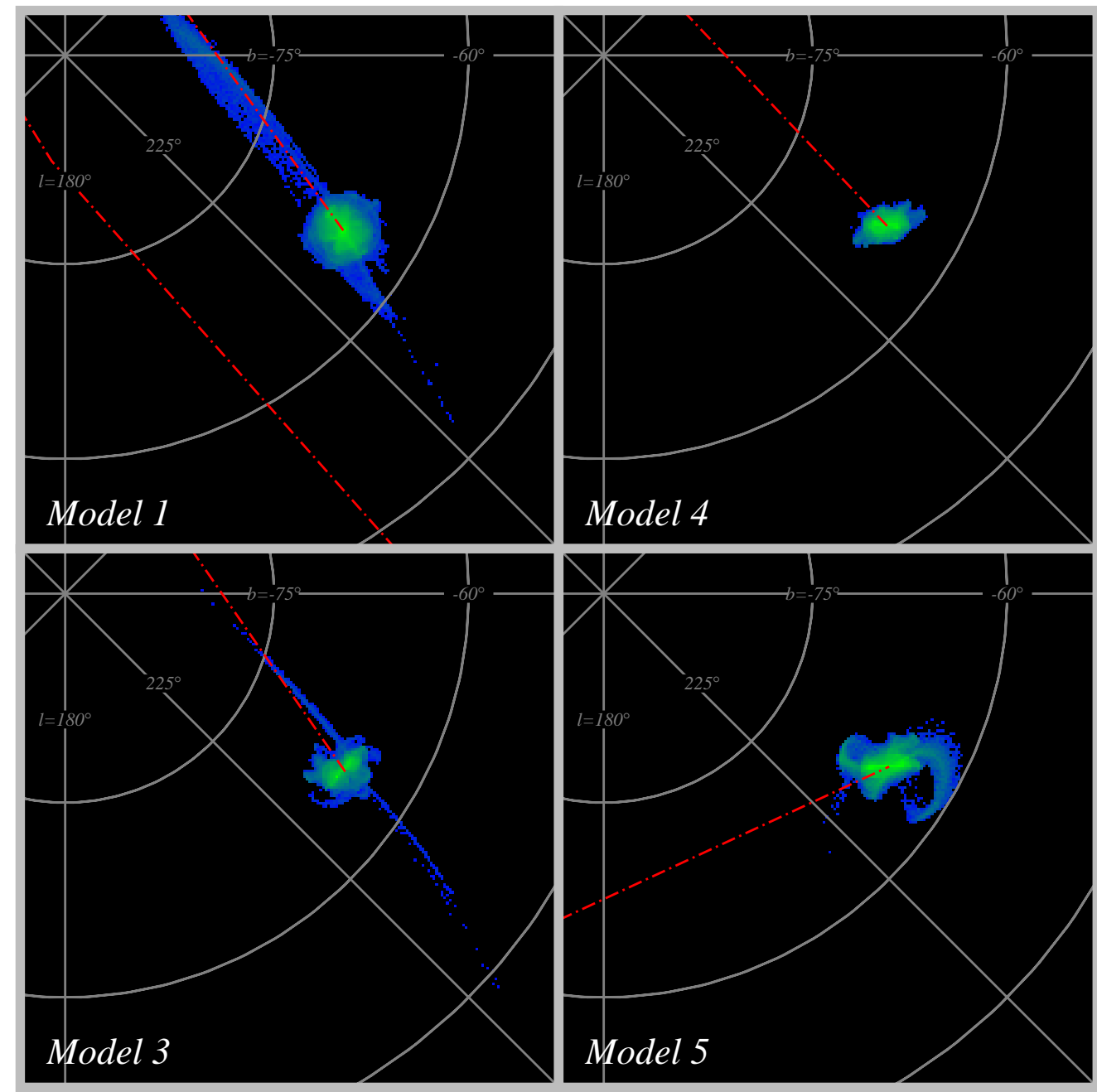

Fig. 4. - The stellar distribution of Fornax $\mathrm{B}$ at present time $(\mathrm{T}=0)$ for Models 1, 3, 4 and 5. The colour scale is the same as used in Figure 2. The orbit of Fornax A is portrayed with the red dashed line. 